\title{
LPA Beamformer for Tracking Nonstationary Accelerated Near-Field Sources
}

\author{
Amira S. Ashour \\ Dept of Electronics \& Electrical Communications Eng., \\ Faculty of Engineering, Tanta Univ., Egypt. Dept of Computer Science, \\ Computer \&IT College, Taif Univ., KSA.
}

\begin{abstract}
In this paper, a computationally very efficient algorithm for direction of arrival (DOA) as well as range parameter estimation is proposed for near-field narrowband nonstationary accelerated moving sources. The proposed algorithm based on the local polynomial approximation (LPA) beamformer, which proves its efficiency with far-field applications. The LPA estimates the instantaneous values of the direction of arrival, angular velocity, acceleration as well as the range parameters of near-field sources using weighted least squares approach which based on Taylor series. The performance efficiency of the LPA beamformer to estimate the DOAs of near-field sources is evaluated and compared with the Recursive Expectation-Maximization (REM) method. The comparison is done using standard deviation of DOA estimation error as well as for range versus signal to noise ratio (SNR). The simulation results show that LPA beamformer outperform REM1 in signal-to-noise ratio requirements.
\end{abstract}

Keywords-Near-field; range and DOA estimation, moving source tracking; LPA beamformer; REM (Recursive Expectation Maximization)

\section{INTRODUCTION}

Direction-of-arrival (DOA) estimation for source localization has been widely applied in the field of radar, sonar, seismology, oceanography and communication. Various algorithms [1], [2] were proposed to deal with the problem of DOA estimation for far-field scenarios. While, near fields are important because they are operationally complex and structurally rich. Away from the antenna, in the far zone, things become predictable; the fields take simple form, and approach plane waves. There is not much to know about the behavior of the antenna aside from the radiation pattern. However, when the sources are located close to the array (i.e. near-field), the wave front emitted from these sources become spherical instead of planar at each sensor position. Therefore, the far-field estimation algorithms are no longer be applicable as the inherent curvature of the waveforms should be taken into account. Therefore, advanced localization algorithms for estimating DOA and range have been derived for near-field stationary source localization, including the modified MUSIC [3], weighted linear prediction (LP) [4], and second-order statistics (SOS) [5, 6]. On the contrary, for localizing a nearfield moving sources of constant velocity, a Stationary Passive Synthetic Aperture Array method was used [7]. Also, the Maximum Likelihood (ML) approach was efficient in case of little sample numbers, but required high computational cost [8]. Therefore, several techniques as Recursive Expectation
Maximization (REM) was studied to reduce the ML complexity. The EM prevents the computational complexity by switching the multidimensional search problem to less dimensional parallel search problems. In [9], the Expectationmaximization (EM) based recursive algorithm was applied with moving sources in the near-field.

Simultaneously, several applications require tracking of accelerated moving sources as the additional use of some kinematic parameters (i.e. acceleration, etc.) to improve the tracking performance and overcomes some of the problems of crossing targets. Consequently, $[10,11]$ have been proposed for the accelerated moving sources in the far-field using the LPA beamformer to localize accelerated moving sources. The LPA proved its perfect performance in different scenarios of fast moving accelerated sources and low SNR. As a result, in this paper the LPA beamformer algorithm is proposed to the scenario of near-field accelerated sources situation. It estimates the time-varying parameters (DOA, angular velocity, acceleration and range) of a near-field moving accelerated sources. The performance of the LPA beamformer is compared with that of the REM1 method using different signal to noise ratios (SNR).

This paper is organized as follows. The problem and data model are formulated in Section II. In Section III, introduce the near-field accelerated sources' parameter estimation algorithm based on the LPA algorithm. In Section IV, the performance analysis and simulation results will presented. Finally, Section V concludes the paper.

\section{PROBLEM Formulation}

Foremost, a description of the radiation zone concept and the time-varying near-field signal model is introduced before establishing the LPA beamformer approach for tracking accelerated time-varying DOA and range parameters of the near-field sources.

\section{A. Radiation Zone}

Radiation zones are defined to simplify the complex mathematical equations for radiating sources. Generally, the radiation zones are divided into the far-field zone and nearfield zone. The wavelength $(\lambda)$ emitted by the source defines the boundary of the two zones. If the distance of the signal emitted by the source to antenna array is large i.e. $r>>\lambda$, then the far-field assumption is valid. In this case, the incoming waves towards the array are approximately planar. Moreover, if the signal emitted by the source is very close to an array, i.e. 
$\mathrm{r}<<\lambda$, then the source is in the near-field region. Therefore, in indoor environments the near-field representation is more suitable with spherical wave fronts than using far-field.

\section{B. Time-varying near-field signal model}

It is assumed that the source signals are collected by $n=2 M+1$ sensors with $d$ distance between its adjacent antennas in a uniform linear array (ULA). As $Q$ narrowband near-field signals from time- varying directions $\theta(t)=\left[\theta_{1}(t), \ldots \ldots, \theta_{Q}(t)\right]$, arrive at the array, the vector $r(t)=\left[r_{1}(t), \ldots \ldots, r_{Q}(t)\right]$ will represent the unknown range parameters of the moving sources. Therefore, the parameter vector to be estimated is $\Theta(t)=\left[\theta^{T}(t), r^{T}(t)\right]^{T}$. Thus, the signal model for the data observed at the output of the sensors at time instant $t$ is,

$$
x(\mathrm{t})=\mathbf{A}(\Theta(t)) \mathbf{s}(\mathrm{t})+\mathbf{e}(\mathrm{t})
$$

The steering matrix which consists of $Q$ steering vectors [ $\left.\mathbf{a}\left(\Theta_{i}(t)\right), \quad i=1, \ldots . . Q\right]$ is,

$$
\mathbf{A}(\Theta(t))=\left[\mathbf{a}\left(\Theta_{1}(t)\right), \mathbf{a}\left(\Theta_{2}(t)\right), \ldots \ldots \ldots \ldots, \mathbf{a}\left(\Theta_{Q}(t)\right)\right]
$$

The steering vector is a function of the unknown parameter vector, $\Theta_{i}(t)=\left[\theta_{i}^{T}(t), r_{i}^{T}(t)\right]^{T}$. Also, $\mathbf{s}(\mathrm{t})$ is the vector of the received signals given by $\mathbf{s}(t)=\left[\mathbf{s}_{1}(t), \ldots \ldots, \mathbf{s}_{Q}(t)\right]$ and the noise vector is $\mathbf{e}(\mathrm{t})=\left[\mathbf{e}_{1}(\mathrm{t}), \mathbf{e}_{2}(\mathrm{t}), \ldots \ldots, \mathbf{e}_{n}(\mathrm{t})\right]$

Similar to [12], the output signal from the $m^{\text {th }}$ element for a given snapshot $t$ is given by

$$
x_{m}(\mathrm{t})=\sum_{q=1}^{Q} e^{j \tau_{m q}} \mathbf{s}_{q}(\mathrm{t})+\mathbf{e}_{m}(\mathrm{t})
$$

Where, $\mathbf{s}_{q}(\mathrm{t})$ is the signal from the $q^{\text {th }}$ incident source, $\mathbf{e}_{m}(\mathrm{t})$ is the noise at the $m^{\text {th }}$ element, and $\tau_{m q}$ is the phase shift associated with a propagation time delay between the element at the center of the array and the sensor $m$ of the $q^{\text {th }}$ source. This phase shift is given by (4) as a function of the source signal parameters, angle of arrival $\theta_{q}$, range $r_{q}$, and the wavelength $\lambda$.

$$
\tau_{m q}=\frac{2 \pi}{\lambda}\left\{\left(\sqrt{\left(\mathrm{r}_{\mathrm{q}}^{2}\right)+(m d)^{2}-2 r_{q} m d \sin \theta_{\mathrm{q}}}\right)-r_{q}\right\}
$$

The $(2 M+1) \times 1$ output vector can be written as $(5)$, where the reference point is the element at the center of the array.

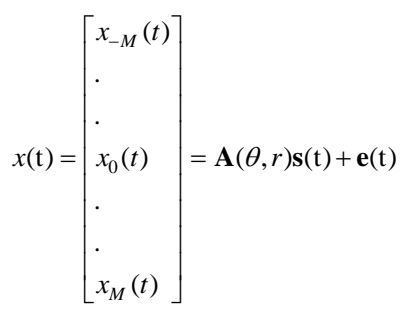

Where, $\mathbf{A}(\theta, r)=\left[\mathbf{a}\left(\theta_{1}, r_{1}\right), \mathbf{a}\left(\theta_{2}, r_{2}\right), \ldots \ldots . \mathbf{a}\left(\theta_{Q}, r_{Q}\right)\right]$ is the $(2 M+1)_{\times}$matrix. As the corresponding array response vector $\mathbf{a}\left(\theta_{q}, r_{q}\right)$ is given by

$$
\mathbf{a}(\Theta)=\mathbf{a}\left(\theta_{q}, r_{q}\right)=\left[\begin{array}{l}
e^{j \tau_{-M q}} \\
\cdot \\
\cdot \\
e^{j \tau_{-q}} \\
\cdot \\
\cdot \\
\cdot \\
e^{j \tau_{M q}}
\end{array}\right]
$$

Approximating $\tau_{m q}$ using the second order Taylor expansion, yields to the following expression for the signal model in (3).

$$
x_{m}(\mathrm{t})=\sum_{q=1}^{Q} e^{j\left(\xi_{q} m+\psi_{q} m^{2}\right)} \mathbf{s}_{q}(\mathrm{t})+\mathbf{e}_{m}(\mathrm{t})
$$

The parameters $\xi_{q}$ and $\psi_{q}$ are functions of the DOA and the range; respectively, and can be expressed as

$$
\xi_{q}=\left(\frac{-2 \pi d}{\lambda} \sin \theta_{q}\right), \quad \psi_{q}=\left(\frac{\pi d^{2}}{\lambda r_{q}} \cos ^{2}\left(\theta_{q}\right)\right)
$$

Therefore, the problem addressed in this paper is the estimate of the DOA's and their parameters (angle, angular velocity and acceleration) as well as the range of incident sources given the observation array data $x(\mathrm{t})$. The LPA beamformer is used to determine the time-varying near-field DOA, range and their parameters under the following assumptions:

1) The source signals are uncorrelated

2) The additive noise is a zero-mean spatially white independent from the source signals

3) The distance $d \leq \lambda_{q}, \lambda_{q}$ is the shortest wavelength of all signals

\section{LPA AlgORITHM For NEAR-FIELd SOURCES' PARAMETER ESTIMATION}

The LPA beamfromer is developed as proposed for accelerated moving near-field sources tracking as its performance advantages with the accelerated moving sources introduced in [10]. It is assumed that the parameters of interest are described by a polynomial model shown in [13]. Using the weighted least squares approach to formulate the LPA beamformer for a single source assumption, which can be extended to the multiple source case. The following LPA based function will be minimized as in $[10,13]$ to be, 


$$
G(t, \Theta)=\frac{1}{\sum_{k} \omega_{h}(k T)} \sum_{k} \omega_{h}(k T)\|x(t+k T)-\mathbf{a}(\Theta, k T) s(t+k T)\|^{2}
$$

Where, $\|\cdot\|$ stands for the norm. The summation interval in (9) is determined by the window function $\omega_{h}(k T)$ which can be given by

$$
\omega_{h}(k T)=\left(\frac{T}{h}\right) \omega\left(\frac{k T}{h}\right)
$$

The scaling parameter $h$ determines the window length, and $\omega(v)$ is a real symmetric function $[\omega(v)=\omega(-v)]$ and satisfies the conventional properties,

$$
\omega(v) \geq 0, \quad \omega(0)=\max _{v} \omega(v), \int_{-\infty}^{\infty} \omega(v) d v=1
$$

The source motion within the observation interval using Taylor series is,

$$
\begin{aligned}
\theta(t+k T)= & \theta(t)+\theta^{(1)}(t)(k T)+\frac{\theta^{(2)}(t)}{2}(k T)^{2}+\frac{\theta^{(3)}(t)}{6}(k T)^{3}+\ldots \\
& =c_{0}+c_{1} k T+c_{2}(k T)^{2}+c_{3}(k T)^{3}+\ldots \ldots
\end{aligned}
$$

Here, $T$ is the sampling interval.

Assuming that the observation window is sufficiently short and, therefore, the fourth and later terms in (12) are negligible, therefore

$$
\theta(t+k T)=c_{0}+c_{1} k T+c_{2}(k T)^{2}
$$

Where,

$$
c_{0}=\theta(t), \mathrm{c}_{1}=\theta^{(1)}(t), \mathrm{c}_{2}=\frac{1}{2} \theta^{(2)}(\mathrm{t})
$$

being the instantaneous source DOA, angular velocity, and acceleration, respectively. So, the problem is to estimate the vector $\mathbf{c}=\left(c_{0}, c_{1}, c_{2}\right)^{T}$ for the nonstationary sources.

Similarly, the range model will be,

$$
r(t+k T)=r_{0}+r_{1} k T+r_{2}(k T)^{2}
$$

Both the DOAs and the ranges can be shown together in the vector, $\Theta(t)=\left[\theta^{T}(t), r^{T}(t)\right]^{T}=\left[\Theta_{1}^{T}, \ldots . ., \Theta_{Q}^{T}\right]^{T}, \quad$ where $\Theta_{q}=\left[c_{0 q}, c_{1 q}, c_{2 q}, r_{0 q}, r_{1 q}, r_{2 q}\right]^{T}$. As $\mathbf{a}(\Theta)$ depends on the time $k T$ and the vectors $\mathbf{c}$ and $r$.

Let us minimize (9) with respect to the unknown deterministic waveform $s(t+k T)$ to get the following,

$\frac{\partial G}{\partial s^{*}(t+k T)}=\frac{-\omega_{h}(k T)}{\sum_{k} \omega_{h}(k T)} \mathbf{a}^{H}(\Theta, k T)\{x(t+k T)-\mathbf{a}(\Theta, k T) s(t+k T)\}=0$

The estimate of the waveform $s(t+k T)$ is obtained as:

$$
\hat{s}(t+k T)=\frac{\mathbf{a}^{H}(\Theta, k T) x(t+k T)}{n}
$$

Recalling that the number of sensors is $n=2 M+1$, the property $\mathbf{a}^{H}(\Theta, k T) \mathbf{a}(\Theta, k T)=n$ is exploited. Inserting (17) into (9) to obtain $G(t, \Theta)$ as,

$G(t, \Theta)=\frac{1}{\sum_{k} \omega_{h}(k T)} \sum_{k} \omega_{h}(k T)\left\{x^{H}(t+k T) x(t+k T)-\frac{\left|\mathbf{a}^{H}(\Theta, k T) x(t+k T)\right|^{2}}{n}\right\}$

Then, minimize (18) over the vector parameter $\Theta$ to obtain the DOA as well as the range parameters. This is equivalent to the maximization of

$$
P(t, \Theta)=\frac{1}{n \sum_{k} \omega_{h}(k T)} \sum_{k} \omega_{h}(k T)\left|\mathbf{a}^{H}(\Theta, k T) x(t+k T)\right|^{2}
$$

where, $|$.$| stands for the absolute value and the function in$ (19) is known as the LPA beamformer function which includes the near-field accelerated moving source parameters. Here, the maximization of the LPA function requires search over $c_{0}, c_{1}, c_{2} r_{0}, r_{1}$ and $r_{2}$.

The steps of the proposed algorithm for near-field parameter estimation is straight forward from that in far- field accelerated sources [10], and can be summarized as follows:

Step1: take initial values of DOA $c^{0}$ and range $r^{0}$ parameters.

Step2: calculate the source motion within the observation interval using Taylor series for the DOAs and range by (13) and (15).

Step3: calculate the LPA beamformer function for DOAs and range in (19).

Step4: Update the parameters.

The same properties of the proposed LPA beamformer with far- field is still applicable in the near-field case, which are:

$>$ The LPA beamformer is convenient for slowly as well as rapidly moving sources.

$>$ The window width selection is important for accurate parameter estimation.

> Adding the acceleration term decreases the MSE.

\section{Simulation Results}

A half wavelength of the incoming signals is used for the spacing between the adjacent elements in the ULA. Uncorrelated near-field narrowband moving sources are assumed with array of $n=9$ sensors. Also, a rectangular window with $\mathrm{N}=50$ snapshots is considered for the LPA beamformer. The following scenarios are used to clarify the LPA beamformer performance in the near-field accelerated moving sources and to compare it with that of REM1 in [9].

Case 1,2: (LPA output for single and double sources) 
Figure 1 shows the LPA beamformer output for near-field single source case in pairs, where the time-varying DOA parameters are $\left[\theta(k), \theta^{(1)}(k), \theta^{(2)}(k)\right]=\left[4^{\circ},-1^{\circ} k, 1^{\circ} k^{2}\right]$.

Similarly, Fig. 2 illustrates two sources scenario in pairs, where the time-varying DOA parameters of the two sources are $\quad\left[\theta_{1}(k), \theta_{1}^{(1)}(k), \theta_{1}^{(2)}(k)\right]=\left[4^{\circ},-2^{\circ} k,-2^{\circ} k^{2}\right] \quad \&$ $\left[\theta_{2}(k), \theta_{2}^{(1)}(k), \theta_{2}^{(2)}(k)\right]=\left[16^{\circ}, 2^{\circ} k, 2^{\circ} k^{2}\right]$.
In is clear that, the LPA can identify correctly the source location, as the peak in each figure indicates the source location at as shown in the figures pairs. The estimates of the DOAs have been obtained from the main maximum of the beamformer function (19). The source acceleration is considered as a factor to improve the source localization in nonstationary situations than using the angle and angular velocity only as in [13].

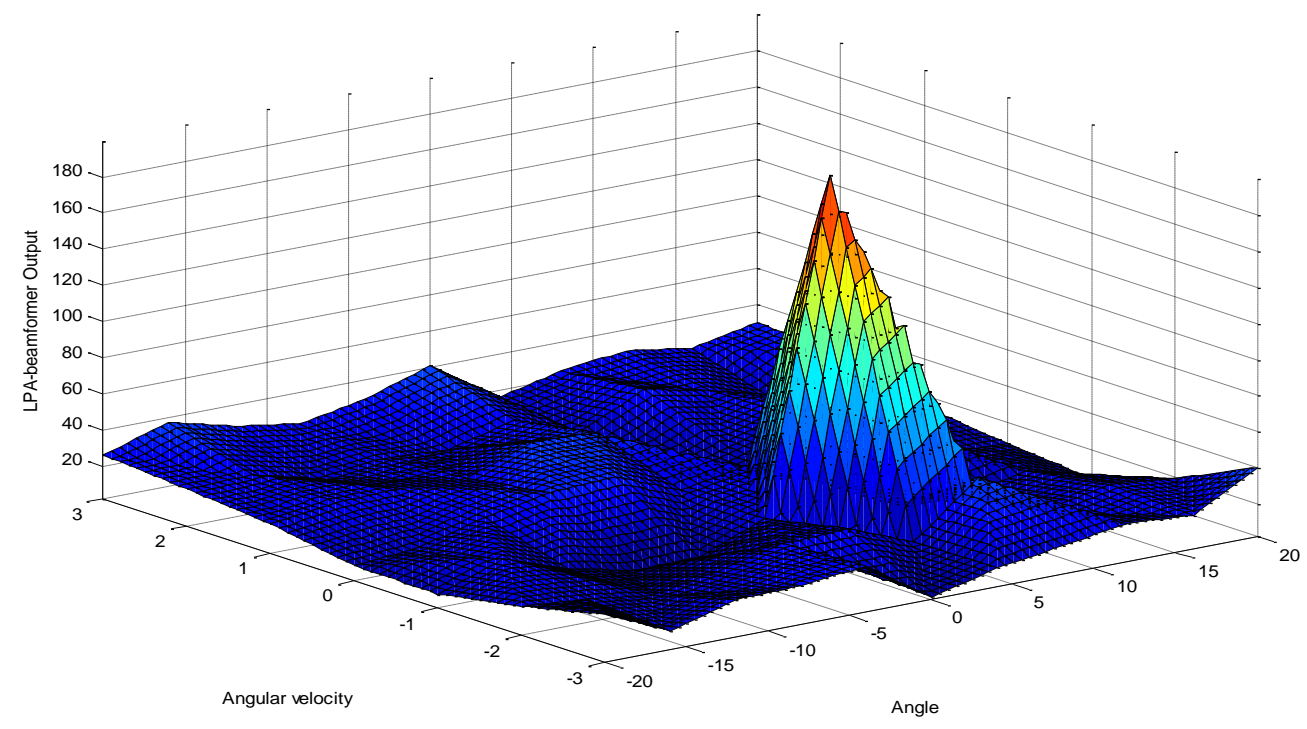

Fig. 1. (a): The output of the LPA beamformer for the single source case, at $5 \mathrm{~dB}$ for $\left(c_{0}, c_{1}\right)$ pair, where the source DOA parameters are:

$\left[\theta(k), \theta^{(1)}(k), \theta^{(2)}(k)\right]=\left[4^{\circ},-1^{\circ} k, 1^{\circ} k^{2}\right]$



Fig. 1. (b): The output of the LPA beamformer for the single source case, at $5 \mathrm{~dB}$ for $\left(c_{0}, c_{2}\right)$ pair, where the source DOA parameters are:

$\left[\theta(k), \theta^{(1)}(k), \theta^{(2)}(k)\right]=\left[4^{\circ},-1^{\circ} k, 1^{\circ} k^{2}\right]$. 


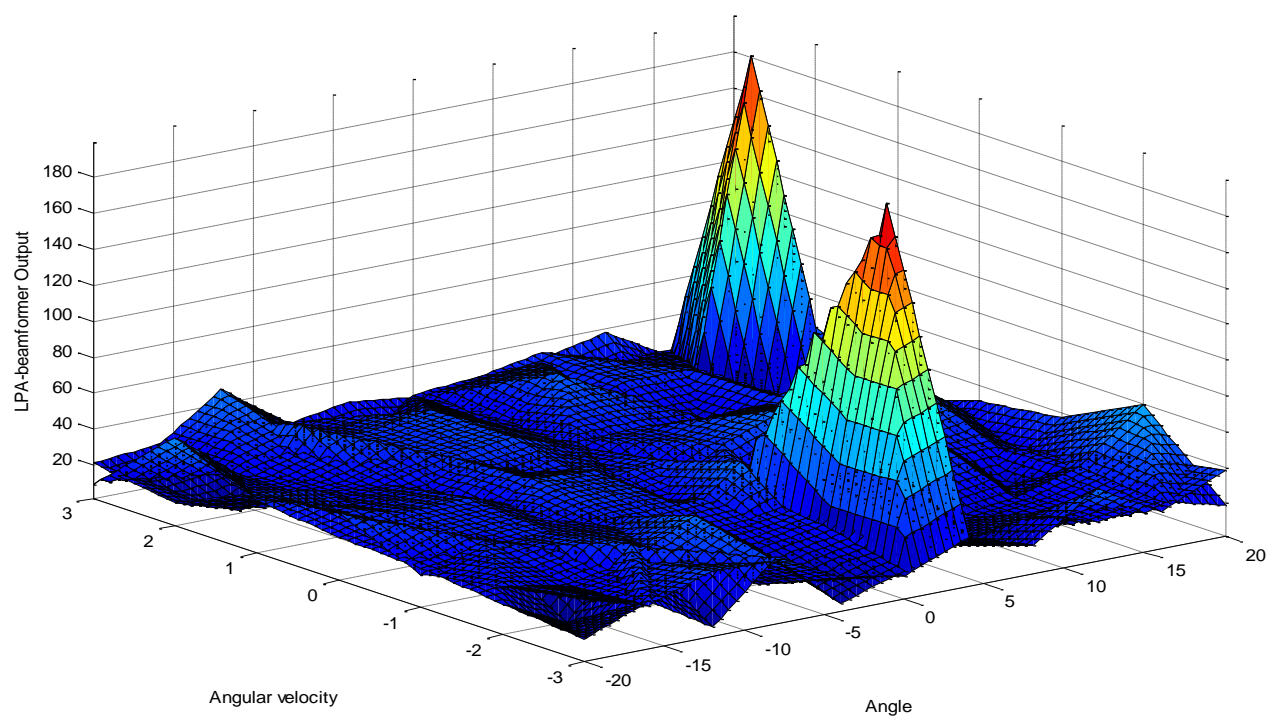

Fig. 2. (a): The output of the LPA beamformer for 2 sources senario, at $5 \mathrm{~dB}$ for $\left(c_{0}, c_{1}\right)$ pair. The DOA parameters of the two sources are:

$\left[\theta_{1}(k), \theta_{1}^{(1)}(k), \theta_{1}^{(2)}(k)\right]=\left[4^{\circ},-2^{\circ} k,-2^{\circ} k^{2}\right]$ and

$\left[\theta_{2}(k), \theta_{2}^{(1)}(k), \theta_{2}^{(2)}(k)\right]=\left[16^{\circ}, 2^{\circ} k, 2^{\circ} k^{2}\right]$.

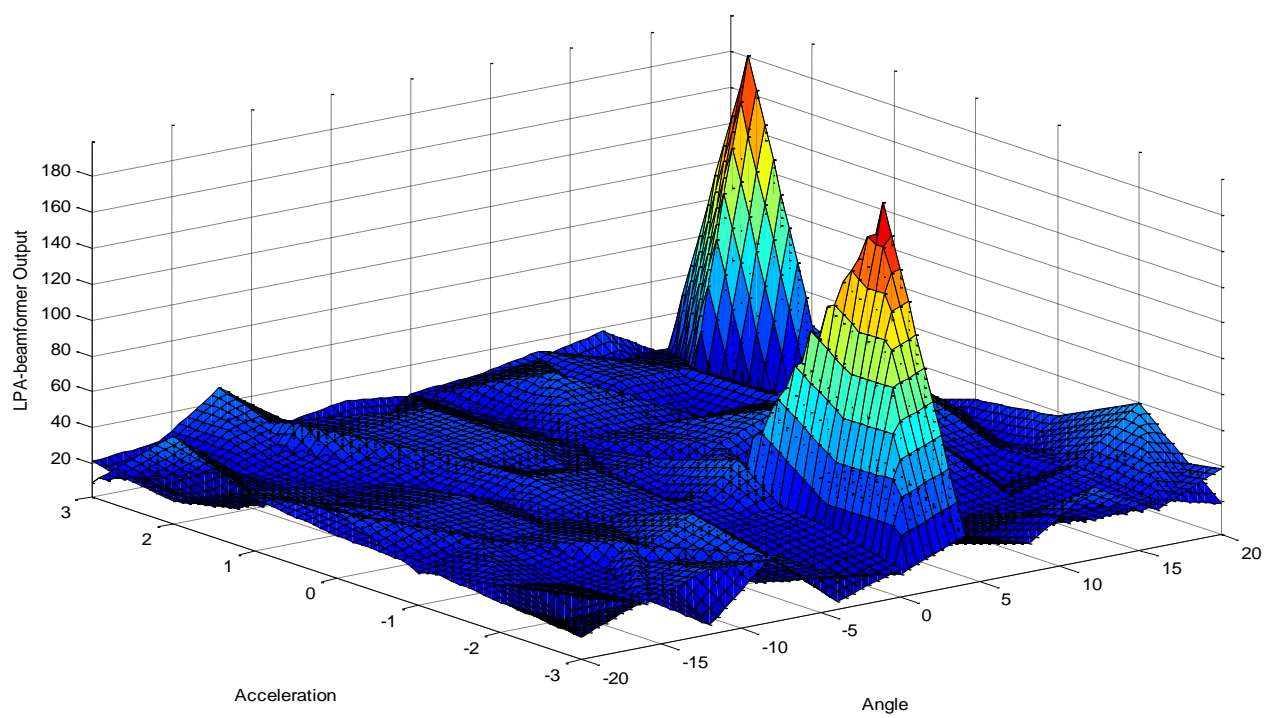

Fig. 2. (b): The output of the LPA beamformer for 2 sources case, at $5 \mathrm{~dB}$ for $\left(c_{0}, c_{2}\right)$ pair. The DOA parameters of the two sources are:

$$
\begin{aligned}
& {\left[\theta_{1}(k), \theta_{1}^{(1)}(k), \theta_{1}^{(2)}(k)\right]=\left[4^{\circ},-2^{\circ} k,-2^{\circ} k^{2}\right] \text { and }} \\
& {\left[\theta_{2}(k), \theta_{2}^{(1)}(k), \theta_{2}^{(2)}(k)\right]=\left[16^{\circ}, 2^{\circ} k, 2^{\circ} k^{2}\right] .}
\end{aligned}
$$

Case 3: (Performance comparison for single source case)

Consider single near-field narrowband accelerated source located at $\left(r_{1}, \theta_{1}\right)=\left(1.5 \lambda, 4^{\circ}\right)$. Figure $3(\mathrm{a}, \mathrm{b})$ shows the standard deviation for DOA and range; respectively, versus SNR for both the LPA beamformer and the REM1 method. The timevarying DOA and range parameter vectors are

$$
\left[\theta_{1}(k), \theta_{1}^{(1)}(k), \theta_{1}^{(2)}(k)\right]=\left[4,-2^{\circ} k, 2^{\circ} k^{2}\right] \quad \text { and }
$$
$\left[r_{1}(k), r_{1}^{(1)}(k), r_{1}^{(2)}(k)\right]=\left[1.5 \lambda,(1 \lambda) k,(1 \lambda) k^{2}\right]$; respectively. It is observed that the proposed method has lower error compared to the REM1 method in the rapidly moving sources scenarios. Both algorithms assume the motion model in (13) and the range model (15). 


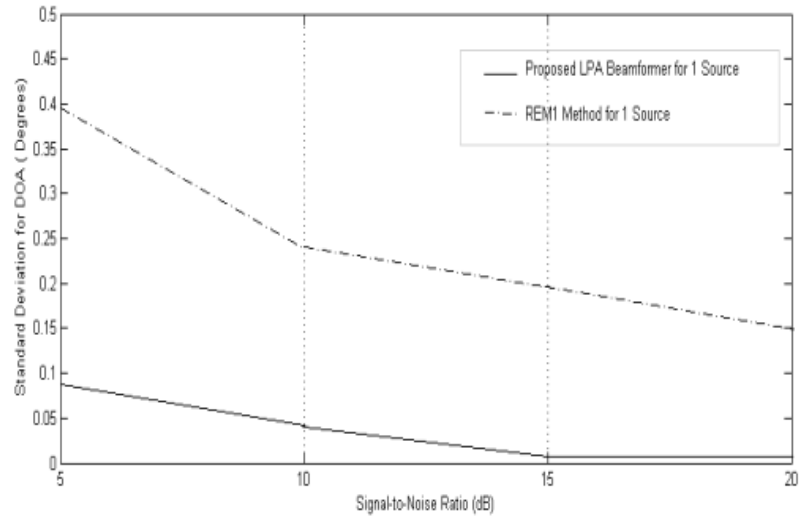

Fig. 3. (a): Compares the standard deviation for DOA angle estimation error versus SNR for a single source that has $\left(r_{1}, \theta_{1}\right)=\left(1.5 \lambda, 4^{\circ}\right)$ for the LPA and the REM1 method.

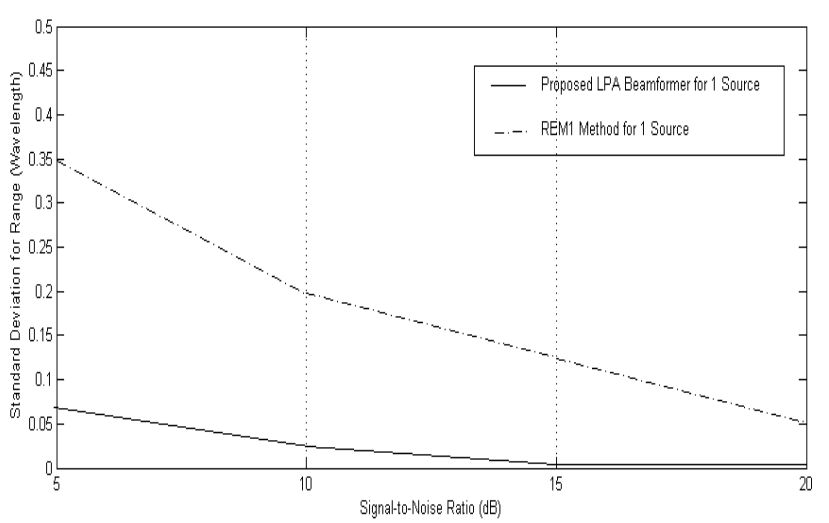

Fig. 3. (b): Compares the standard deviation for range estimation error versus SNR for a single source that has $\left(r_{1}, \theta_{1}\right)=\left(1.5 \lambda, 4^{\circ}\right)$ for the LPA algorithm and the REM1 method.

Case 4 :( Performance comparison for Two sources case)

Assume two near-field uncorrelated narrowband moving sources located at $\left(r_{1}, \theta_{1}\right)=\left(2 \lambda, 4^{\circ}\right)$ and $\left(r_{2}, \theta_{2}\right)=\left(4 \lambda, 16^{\circ}\right)$. The time-varying DOA parameters for the two sources is $\left[\theta_{1}(k), \theta_{1}^{(1)}(k), \theta_{1}^{(2)}(k)\right]=\left[4,-2^{\circ} k, 2^{\circ} k^{2}\right]$

$\left[\theta_{2}(k), \theta_{2}^{(1)}(k), \theta_{2}^{(2)}(k)\right]=\left[16,2^{\circ} k,-2^{\circ} k^{2}\right]$.

While the range parameters for the two sources are:

$$
\begin{aligned}
& {\left[r_{1}(k), r_{1}^{(1)}(k), r_{1}^{(2)}(k)\right]=\left[2 \lambda,(1 \lambda) k,(1 \lambda) k^{2}\right],} \\
& {\left[r_{1}(k), r_{1}^{(1)}(k), r_{1}^{(2)}(k)\right]=\left[2 \lambda,(1 \lambda) k,(1 \lambda) k^{2}\right] .}
\end{aligned}
$$

It is clear from Fig. 4 that the proposed LPA beamformer is superior to the REM1 method.

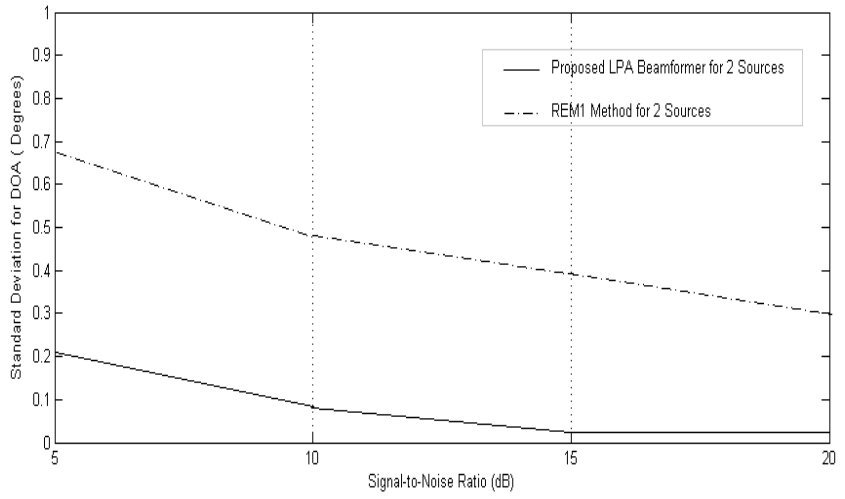

Fig. 4. (a): Compares the standard deviation for DOA angle estimation error versus SNR for 2 sources located at $\left(r_{1}, \theta_{1}\right)=\left(2 \lambda, 4^{\circ}\right)$ and $\left(r_{2}, \theta_{2}\right)=\left(4 \lambda, 16^{\circ}\right)$ for the proposed algorithm and the REM1 method.

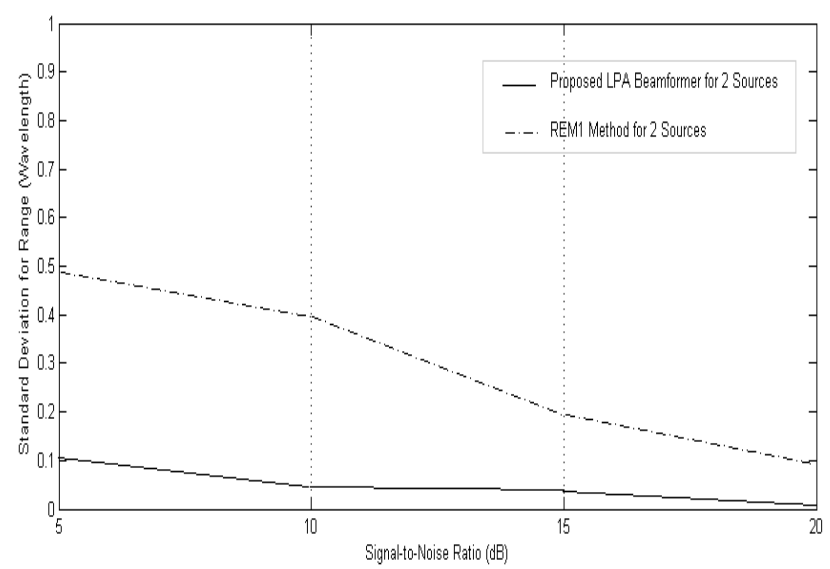

Fig. 4. (b): Compares the standard deviation for range estimation error versus SNR for 2 sources located at $\left(r_{1}, \theta_{1}\right)=\left(2 \lambda, 4^{\circ}\right)$ and $\left(r_{2}, \theta_{2}\right)=\left(4 \lambda, 16^{\circ}\right)$ for the proposed algorithm and the REM1 method.

\section{CONCLUSION}

The proposed method represented more accurately the near-field scenarios, typical of indoor environments. Where, in this paper an efficient nonparametric approach based on LPA beamformer for DOA and range parameters estimation for near-field moving source tracking is proposed. By exploiting the acceleration of the moving source, the standard deviation of the angle as well as range estimation using LPA beamformer is less than compared to the REM1. The simulations show that, this proposed nonparametric technique has superior performance over the REM1 method. These advantages appropriate the proposed algorithm for rapidly moving sources. The simulation verifies the effectiveness of the LPA beamformer with the near-field scenarios.

REFERENCES

[1] R. Schmidt, "Multiple emitter location and signal parameter estimation," IEEE Trans. Antennas Propagation, Vol. 34, No. 3, pp. 276-280, 1986.

[2] R. Roy and T. Kailath, "ESPRIT-estimation of signal parameters via rotational invariance techniques," IEEE Trans. Acoustic, Speech and Signal Process, Vol. 37, No. 7, pp. 984-995, 1989. 
[3] Y. Huang and M. Barkat, "Near-field multiple source localization by passive sensor array, "IEEE Transactions on Antennas and Propagation, Vol. 39, pp. 968-975, 1991.

[4] E. Grosicki, K. Abed-Meraim and Y. Hua, "A weighted linear prediction method for near-field source localization," IEEE Trans. Signal Processing, Vol. 53, pp. 3651-3660, 2005.

[5] R. Boyer and J. Picheral, "Second-order near-field localization with automatic paring operation," ICASSP, pp. 2569-2572, 2008.

[6] X. Qian, L. Hong, “ A Novel Near-Field Source Localization Algorithm," 2nd International Conference on Signal Processing Systems (ICSPS), Vol.2, pp.188-191, 2010.

[7] Z. Wang, F. Tian, Y. Yang and L. Xu, "Moving Source Localization in Near-field by a Stationary Passive Synthetic Aperture Array," APSIPA ASC, 2011 .

[8] N. Kabaoğlu, H.A. Çırpan, S. Paker, "Unconditional Maximum Likelihood Approach for Localization of Near-Field Sources in 3D Space," Fourth IEEE International Symp. on Signal Processing and Information Technology, pp. 233-237, 2004.

[9] S. Cekli, E. Cekli, N. Kabaoglu and H.A. Cirpan, "EM-Based Recursive Tracking Algorithm for Near-Field Moving Sources," IEEE Intelligent Vehicles Symposium Conference. pp. 381-385, 2007.

[10] H.M. Elkamchouchi, M.E. Nasr, A.S. Ashour, "Modified LPA beamformer for localizing and tracking rapidly accelerated moving sources," NRSC B4,pp. 1-8, 2004.

[11] H.M. Elkamchouchi, M.E. Nasr, A.S. Ashour, " Nonparametric approach for direction-of-arrival estimation of accelerated moving sources using cubic array," Alexandria Engineering JournalAEJ, 2005.

[12] N. Kabaoglu, H.A. Cirpan, S. Paker, "EM Based Stochastic Maximum Likelihood Approach for Localization of Near-Field Sources in 3-D," Frequenz, Journal of Telecommunications, 2004, Vol. 58, pp. 178-184, 2004.

[13] V. Katkovnik and A .B. Gershman, "Performance Study of the Local Polynomial Approximation Based Beamforming in the Presence of Moving Sources," IEEE Trans. on Antennas and Propagation, Vol.50, pp. 1151-1157, 2002.

\section{AUTHORS PROFILES}

Amira Ashour, B.Sc in electrical engineering, 1997. The M.Sc in "Enhancement of Electromagnetic Non-Destructive Evaluation Performance Using Advanced Signal Processing Techniques," Faculty of Engineering, Egypt, 2000. She has been awarded the Ph.D degree in Electronic and Communication Engineering in the field of Smart Antenna, Faculty of Engineering, Tanta University, Egypt, 2005. She is now the head of the Computer Science Department, College of Computers and Information Technology, Taif University, Kingdom of Saudi Arabia. She has papers in the field of smart antenna, adaptive antenna arrays and signal processing. 